\title{
Plasmodium falciparum genetic factors rather than the hosts' are likely to drive resistance to acts in ghana.
}

\section{Peter Hodoameda}

University of Ghana College of Basic and Applied Sciences

\section{Nancy Duah Quashie}

University of Ghana Noguchi Memorial Institute for Medical Research

Charles Oheneba Hagan

University of Cape Coast School of Medical Sciences

\section{Sena Matrevi}

University of Ghana Noguchi Memorial Institute for Medical Research

Benjamin Abuaku

University of Ghana Noguchi Memorial Institute for Medical Research

\section{Kwadwo Koram}

University of Ghana Noguchi Memorial Institute for Medical Research

\section{Neils B Quashie ( $\square$ nquashie@noguchi.ug.edu.gh )}

University of Ghana School of Medicine and Dentistry

\section{Research}

Keywords: Anti-malarial drug resistance, Cytochrome 3A4 (CYP3A4), Cytochrome 2C8 (CYP2C8), multidrug resistance, Artesunate, Amodiaquine, Artemether lumefantrine, Molecular markers, Mutations, Ghana

Posted Date: January 21st, 2020

DOl: https://doi.org/10.21203/rs.2.21377/v1

License: (1) This work is licensed under a Creative Commons Attribution 4.0 International License. Read Full License

Version of Record: A version of this preprint was published at Malaria Journal on July 15th, 2020. See the published version at https://doi.org/10.1186/s12936-020-03320-7. 


\section{Abstract}

Background: Artemisinin-based Combination Therapy (ACT), a combination of a fast-acting artemisinin derivative and a relatively slow-acting partner drug, is used for malaria treatment in disease-endemic areas. The ACT partner drugs, currently used in Ghana are lumefantrine, amodiaquine, and piperaquine. Plasmodium falciparum isolates with reduced susceptibility to these partner drugs may affect treatment outcome. Mutations in the parasite multidrug-resistant 1 (Pfmdr1) gene is linked to reduced susceptibility to amodiaquine and lumefantrine. In addition, the potency of the partner drugs in vivo depends on the metabolism by the cytochrome P450 (CYP) enzyme in the host. Mutations in the CYP2C8 gene are linked to reduced metabolism of amodiaquine in vitro while mutations in CYP3A4 may be linked to reduced metabolism to lumefantrine in vitro.

Methods: This study investigated the host and parasite genetic factors affecting the susceptibility of the malaria parasite to ACT partner drugs. Archived samples from 240 patients (120 given AL and the other half given $A A$ ) aged $\leq 9$ years participating in antimalarial drug resistance survey in sites representing the three ecological areas of Ghana were used. Polymerase chain reaction (PCR) followed by Sanger sequencing was used to determine the polymorphisms in CYP2C8, CYP3A4, and Pfmdr1 genes.

Results: Of the 93 samples successfully genotyped for CYP3A4, all had wild type alleles which suggest that the hosts are good metabolizers of lumefantrine. Ninety-four samples were successfully genotyped for CYP2C8 of which $60 \%$ had wild type alleles, $35 \%$ heterozygous and $5 \%$ homozygous recessive alleles. The high percentage of wild type alleles observed also suggests that amodiaquine was metabolized efficiently by the hosts. Ninety-five samples were successfully genotyped for the Pfmdr1 gene. At codon $86,95 \%$ were wild type (N86), $5 \%$ mutant (Y86). For codon 184, 36\% were wild type (Y184) and 64\% mutant (F184) while for codons 1034, 1042 and 1246, 100\% (all) were wild type. The high prevalence of N86, F184, and D1246 (NFD) suggest the presence of strains of the parasites with reduced susceptibility to lumefantrine and not amodiaquine. Delayed clearance was observed in individuals with mutations in the Pfmdr 1 gene and not Cytochrome 450 gene. There were both synonymous and nonsynonymous mutations observed in the Pfmdr1 at low prevalence.

Conclusion: The outcome of this study indicates that parasite's genetic factors rather than the hosts' are likely to drive resistance to ACTs in Ghana.

\section{Background}

Malaria is one of the major causes of morbidity and mortality in sub-Saharan Africa, especially in children under 5 years and pregnant women (WHO, 2018). WHO recommends the use of a combination of a fast-acting artemisinin derivative and a relatively slow-acting partner drug, for malaria treatment in disease-endemic areas (WHO, 2014). The recommended first line ACTs in Ghana for treating 
uncomplicated malaria are artesunate with amodiaquine (AA), artemether with lumefantrine (AL) or a combination of Dihydroartemisinin with piperaquine (Ministry of Health, 2009).

The reason for combining the drugs (ACT) is to slow down the development of resistance to the antimalarial drugs by $P$. falciparum (White \& Nosten, 2007). The fast acting drug quickly reduces the parasite load whilst the slow acting antimalarial gradually mob up the residue parasites. The potency of the ACTs is dependent on the efficacy of both the artemisinin component and the partner drug (White \& Nosten, 2007). Reduced susceptibility of parasites to the partner drugs in the ACTs can therefore potentially result in resistance to artemisinin in future as parasites that escape the fast action of the artemisinin or its derivatives will not be cleared by the partner drugs and this could allow ample time for growth and expansion of 'drug resistant parasite population' (White \& Nosten, 2007).

The variations observed in the effectiveness of ACTs in malaria-endemic regions is dependent on the parasite genetic factors (Ouji et al., 2018), as well as the human genetic factors (Zanger \& Schwab, 2013). For the parasite genetic factors, polymorphisms which arise due to single nucleotide changes in the Pfmdr1 gene in its coding region have been linked to differential parasite susceptibility to the ACT partner drugs, like amodiaquine (Sá et al., 2009) and lumefantrine (Sisowath et al., 2007). This makes Pfmdr1 an important likely candidate for initiating ACT partner drug resistance (Chen et al., 2010). The polymorphic $P f m d r 1$ alleles that are mostly found in Africa are N86Y, F184Y, and D1246Y. The $P$. falciparum N86-F184-D1246 haplotype (NFD haplotype) has been linked to decreased susceptibility to antimalarial drugs such as mefloquine and lumefantrine. The selection of the NFD haplotype has been seen in malaria treatment using artemether+lumefantrine. The different haplotype which is the Y86-Y184Y1246 haplotype (YYY haplotype) is associated with reduced amodiaquine susceptibility (Holmgren et al., 2007).

Differences in the genetic make-up of humans are the principal factor that defines the level of drug availability in the blood to clear the parasites (Zanger \& Schwab, 2013). The cytochrome P450 enzyme family (CYP genes) is involved in the metabolism of the different antimalarial drugs (Zanger \& Schwab, 2013). Amodiaquine is mainly metabolized by CYP2C8 (Parikh et al., 2007) whiles Lumefantrine is metabolized mainly by CYP3A4 (Lee et al., 2012). Different mutations in the promoter region, introns or exons can result in different alleles of the CYP450 genes in different individuals. The metabolism of a drug or a combination of drugs could be decreased, increased or unaffected depending on the allele(s) an individual possesses ( $\mathrm{Wu}, 2011)$. Elucidating the exact role these disparities in the genes coding for the enzymes involved in ACTs metabolism is vital for understanding the inter-individual pharmacokinetic differences observed in persons using ACTs (Ingelman-Sundberg \& Rodriguez-Antona, 2005). This study investigated $P$. falciparum and host genetic factors that are likely to affect the efficacy of the ACT partner drugs used in Ghana.

\section{Methods}

Study sites 
Three sentinel sites, Navrongo, Begoro and Cape Coast which represent the three distinct ecoepidemiological zones in Ghana were selected for this study (Figure 1). Begoro is located in the tropical forest ecological zone, Navrongo is in the Northern Savanna ecological zone and Cape-Coast is situated in the Coastal Savanna ecological zone.

\section{Study Samples}

Blood blot filter paper used in this study were archived samples previously taken from children who have been diagnosed with uncomplicated $P$. falciparum malaria and met the inclusion criteria after an informed consent has been obtained from their parents or guardian. A medical doctor prescribes either AA or AL to the study participants who were then followed up for 28 days. The archived samples from a group of the participants referred hereafter as 'cohort 1' comprises of 120 patients who were given AL and a second group, cohort 2, comprising of 120 patients were given AA. Of the 240 study participants, 60 were selected from the Savannah zone, 90 from the Coastal zone and 90 from the Forest zone.

\section{Detection of Pfmdr1 polymorphisms}

A total of 95 out of 120 samples were successfully analyzed for Pfmdr 1 polymorphisms. The polymorphisms were analysed at codons 86 (asparagine to tyrosine), 184 (tyrosine to phenylalanine), 1034 (serine to cysteine), 1042 (asparagine to aspartic acid) and 1246 (aspartic acid to tyrosine). A polymerase chain reaction followed by Sanger sequencing was used in determining these polymorphisms as previously described (Vinayak et al., 2010).

\section{Detection of CYP2C8 and CYP3A4 polymorphisms}

A total of 94 samples out of 120 were successfully analyzed for CYP2C8 polymorphisms while 93 out of 120 was successfully analyzed for CYP3A4 polymorphisms. The CYP2C8 polymorphisms were analysed at codon 269 (Isoleucine to phenylalanine). The CYP3A4 polymorphisms were analysed at position $392 A>G$ of the proximal promoter region. A polymerase chain reaction followed by Sanger sequencing was used in determining the polymorphism in CYP2C8 as reported by the group of Cavaco (Cavaco et al., 2005) and CYP3A4 as described by Hodel and colleagues (Hodel et al., 2009).

\section{Statistical Analysis}

Data were analyzed using R software, SPSS software (version 20) and GraphPad Prism version 6. Genotype deviations from the Hardy-Weinberg equilibrium were also determined. All tests were considered statistically significant when $p$-value $<0.005$.

\section{Results}

Prevalence of individuals CYP3A4 and CYP2C8 genotype 
Ninety-three (93) individuals were successfully genotyped for CYP3A4 of which all (100\%) were wild type. The genotypes analyzed for CYP3A4 was not in Hardy-Weinberg equilibrium. Ninety-four (94) individuals were successfully genotyped for CYP2C8 of which 60\% (56/94) had wild type alleles, 35\% (33/94) heterozygous and 5\% (5/94) homozygous recessive alleles. The genotypes analyzed for CYP2C8 was in Hardy-Weinberg equilibrium.

Prevalence of isolates with Pfmdr1 codons 86, 184, 1034, 1042, 1246 alleles and NFD haplotype

Ninty-five $P$. falciparum out of 120 clinical isolates was successfully genotyped for the $P f m d r 1$ gene. For Pfmdr1 genotype at codon 86, 95\% (90/95) were wild type (N86) and 5\% (5/95) mutant (Y86). For codon 184, 36\% (34/95) were wild type (Y184), 64\% (61/95) mutant (F184) while for codons 1034, 1042 and 1246 , all (100\%) were wild type. There were both nonsynonymous and synonymous mutations observed at low frequencies in some of the samples analyzed. The Pfmdr1 haplotypes observed were $57.8 \%$ (55/95) NFD, 34.7\% (33/95) NYD, 6.3\% (6/95) YFD and 1\% (1/95) YYD.

Table 1 Novel synonymous and non-synonymous mutations at coastal and savannah Ecological zones

\begin{tabular}{|lll|}
\hline $\begin{array}{l}\text { Ecological } \\
\text { Zone }\end{array}$ & $\begin{array}{l}\text { Novel synonymous mutation [frequency in } \\
\text { parenthesis] }\end{array}$ & $\begin{array}{l}\text { Novel Non-synonymous mutation } \\
\text { [frequency in parenthesis] }\end{array}$ \\
\hline Coastal & $\begin{array}{l}\text { F106S [1], E236K [1], Y296N [1], E275K [1], } \\
\text { E261K [1], S1217Y [1]. }\end{array}$ & $\begin{array}{l}\text { G293G [1], G102G [1], G284G [1], I1119I } \\
\text { [1], L127L [1]. }\end{array}$ \\
\hline Savannah & E236K [1], E275K [3], R299K [2], E261K [3]. & L108L [1], D117D [1], T1069T [1]. \\
\hline
\end{tabular}

Six novel synonymous mutations were observed in the Coastal zone and 9 in the Savannah zone. Multiply synonymous mutations were observed in samples from Savannah zone. With regard to the novel non-synonymous mutations, 5 were observed in samples from the Coastal zone whilst 3 were seen in samples from the Savannah zone

Table 2 CYP3A4 wildtype individuals for lumefantrine metabolism and parasite Pfmdr1 mutation(s) among those with delayed parasite clearance (day 3 positive) 


\begin{tabular}{|lll|}
\hline Sample number & CYP3A4 Genotype & Pfmdr1 mutation (s) \\
\hline 1 & AA & F184 \\
\hline 2 & AA & F184 \\
\hline 4 & AA & F184 \\
\hline 5 & AA & F184 \\
\hline 6 & AA & F184 \\
\hline 7 & AA & F184 \\
\hline 8 & AA & F184 \\
\hline 9 & AA & Y86, F184 \\
\hline 10 & AA & F184 \\
\hline 11 & AA & F184 \\
\hline 12 & AA & F184 \\
\hline 13 & AA & F184 \\
\hline 14 & AA & F184 \\
\hline 15 & AA & F184 \\
\hline 16 & AA & F184 \\
\hline & AA & F184 \\
\hline
\end{tabular}

Sixteen of the samples with delayed clearance had the CYP3A4 wild type individuals for lumefantrine metabolism and parasite Pfmdr1 mutation(s) as indicated

Table 3 CYP2C8 wildtype and heterozygous individuals for amodiaquine metabolism and parasite Pfmdr1 mutation(s) among those with delayed parasite clearance (day 3 positive)

\begin{tabular}{|lll|}
\hline Sample number & CYP2C8 Genotype & Pfmdr1 mutation (s) \\
\hline 1 & AA & F184 \\
\hline 2 & AA & F184 \\
\hline 3 & AT & F184 \\
\hline 4 & AT & Y86 \\
\hline 5 & AT & Y86, F184 \\
\hline
\end{tabular}




\section{Discussion}

It is predictable from the aforementioned mechanism justifying the use of ACT for malaria treatment that resistance of $P$. falciparum to the ACT partner drugs may lead to the gradual selection of strains of the parasites with reduced susceptibility to the artemisinins. The failure of the partner drugs should therefore be of great concern to the WHO and the National Malaria Control Program in disease endemic areas. As shown in Table 2 and 3,13\% (16/120) of the patients on AL (cohort 1) still carried parasites on day 3 post-treatment compared to $4 \%(5 / 120)$ of those on AA (cohort 2). However all parasites were cleared by day 7 post treatment. This observation, indicate a better rate of parasite clearance with $A A$ than $A L$ and gives credence to the clinical outcome of the study already reported by Abuaku and colleagues, (Abuaku et al., 2016) who are the original owners of the samples used in this study.

The efficacy of the partner drugs investigated in this study, amodiaquine and lumifantrine, is related to Pfmdr1 gene which is part of the ATP-Binding Cassette (ABC) transporters (Ferreira et al., 2011). This gene encodes a transporter which is found in the digestive vacuole (Bopp et al., 2018). The Pfmdr1 function by pumping compounds out of the parasite thus making it an important protein for antimalarial drug resistance. Despite this knowledge, it must be still emphasized that the true mechanistic role of the Pfmdr1 in initiating antimalarial drug resistance is poorly understood (Chen et al., 2010) although certain mutations in the gene have been associated with resistance to different antimalarial drugs (Sá et al., 2009; Sisowath et al., 2007). Polymorphisms in the Pfmdr1 have been linked to differential susceptibility to amodiaquine (Sá et al., 2009) and lumefantrine (Sisowath et al., 2007). The polymorphic Pfmdr1 alleles mostly found in Africa are N86Y, F184Y, and D1246Y. The $P$. falciparum NFD haplotype is associated with decreased susceptibility to lumefantrine. The selection of the NFD haplotype has been seen in malaria treatment using artemether with lumefantrine. The different haplotype which is the YYY haplotype is associated with reduced susceptibility to amodiaquine (Holmgren et al., 2007). From the results of this study, Pfmdr1 codon 86 alleles ( $N$ or $\mathrm{Y}$ ), codon 184 alleles ( $\mathrm{Y}$ or $\mathrm{F}$ ) and codon 1246 alleles (D or $Y$ ) were detected in 95 samples. Of this, 25 (26\%) samples were collected from Navrongo, 39 (41\%) from Begoro (36\%), and 31 (33\%) from Cape Coast. This indicate that the presence of these mutation is wide spread in Ghana and is not ecological zonal bias. For the Pfmdr1 codon 86 ( $\mathrm{N}$ or $\mathrm{Y}$ allele), the prevalence of the mutant allele Y86 for all the individual sites ranged from 12\% (3/25) in Navrongo, 5.5\% $(2 / 39)$ in Begoro, and 6.5\% (2/31) in Cape Coast. All put together this observation indicate a high prevalence of the wild type allele, N86. For the Pfmdr1 codon 184 alleles $(\mathrm{Y}$ or $\mathrm{F})$, the prevalence of the mutant allele F184 observed were 72\% (18/25) in Navrongo, 59\% (23/39) in Begoro and 64.5\% (20/31) in Cape Coast. For the Pfmdr1 codon 1246 (D or Y allele), the prevalence of the wildtype allele D1246 was $100 \%$ for all the sites. High prevalence of N86, F184, and D1246 haplotypes were observed in this study with no record of Y86, Y184 and Y1246 haplotypes. The high prevalence of the NFD haplotype recorded in this study is consistent with that reported by Duah and colleagues (Duah et al., (2013). Although the results show some consistency with the day 3 treatment outcome, however, since in vitro test of parasite susceptibilities to lumefantrine and amodiaquine were not performed in this study the association between $P f m d r 1$ haplotypeamplification and resistance to the antimalarial drugs could be difficult to established. 
There were similar numbers of both non-synonymous and synonymous mutations observed at low frequencies in the coastal and forest ecological zones. The synonymous mutations may not have any significant effect on the susceptibility of the parasite to the antimalarial drugs since it does not lead to change in amino acids. The novel non-synonymous mutations observed in this study may suggest the possible emergence of new mutations that may lead to reduced parasites susceptibility to ACTs in Ghana.

The enzymatic biotransformation of a drug to its active metabolite or bio activation to the therapeutically relevant molecule is vital in order to be effective against its target (Kebamo et al., 2015). Variation in the genetic make-up of humans is the principal factor that defines the level of drugs available in mostly the blood to clear the parasite. The cytochrome P450 enzyme family (CYP genes) is a key enzyme involved in different antimalarial drug metabolism available for the treatment of malaria (Zanger \& Schwab, 2013). Lumefantrine is metabolized to desbutyl-benflumetol mainly by CYP3A4 (Lefevre \& Thomseadn, 1999). Mutation in the gene proximal promoter region which results from a change from adenine (A) to guanine (G) at the position 392 results in CYP3A4*1B (Lamba, Lin, Schuetz, \& Thummel, 2012) which have been suggested to have poor enzyme activity (Mutagonda et al., 2017). From the results of the current study, 93 individuals were successfully genotyped for CYP3A4 of which $100 \%$ were wild type. This result is contrary to what was reported by Kudzi and colleagues (Kudzi et al., 2010). The high number of individuals with wild type CYP3A4 suggests that lumefantrine is well metabolized in the participants. Again, delayed clearance observed in patients treated with AL were seen to have one or more mutations in the Pfmdr1 gene of the P. falciparum clinical isolates and no mutation in the CYP3A4 gene of the individuals. From this, it can be inferred that the parasite genetic factors are likely to be the cause of delayed clearance in the children treated with AL. A limitation of this study is the absence of desbutyllumefantrine pharmacokinetic data of the study participants to film some of our assertions. Nonetheless, these results are in agreement with findings from Kiaco et al., (2017).

The CYP2C8 is the main enzyme that metabolizes amodiaquine to desethyl amodiaquine (DEAQ) (Li et al., 2002). The wild type $C Y P 2 C 8 * 1$ and the mutant $C Y P 2 C 8 * 2$ are the most predominant in Ghana (Kudzi et al., 2009). A change from adenine (A) to thymine ( $T$ ) at nucleotide position 895 on exon 5 results in the CYP2C8*2 mutant. CYP2C $8 * 2$ has been shown to be associated with decreased enzyme activity in vitro and reduced intrinsic clearance of amodiaquine (Parikh et al., 2007). From the results of the study, 94 individuals were successfully genotyped for CYP2C8 of which $60 \%(56 / 94)$ had wild type alleles, $35 \%$ (33/94) heterozygous and 5\% (5/94) homozygous recessive alleles. This result is contrary to what has been reported by Kudzi et al., (2009). The high number of individuals with wild type CYP2C8 suggests that amodiaquine was well metabolized in the participants. It must however be emphasized that delayed clearance was observed in individuals who reported with high parasitemia (parasitemia $>100,000$ ) on day 0 and with one or more mutation(s) in the Pfmdr1 gene. These individuals had either wild type or heterozygous CYP2C8 genotype suggesting ample concentration of DEAQ in the plasma. Thus it was expected that their parasites should have been easily cleared. There was no delayed clearance observed in $C Y P 2 C 8 * 2$ individuals. This may imply that the CYP2C8 genotype of an individual may not alter the metabolism of the drug significantly, hence the plasma concentration of DEAQ may be adequate to clear 
the parasite. It must be noted that this study could not provide direct evidence to support the aforementioned phenomenon. The absence of delayed clearance in $C Y P 2 C 8 * 2$ individuals can also be explained by the fact that dihydroartemisinin (DHA) which is a metabolite of artesunate in the patients clears most of the parasites and leaves only a few supposedly 'weakened parasite' residues making the presence of a suboptimal concentration of DEAQ enough to clear the parasite residue in these individuals.

\section{Conclusion}

In conclusion, all individuals successfully genotyped for CYP3A4 were wild type, suggesting that lumefantrine is well metabolized in the participants. For CYP2C8, 60\% (56/94) had wild type alleles, 35\% (33/94) heterozygous and 5\% (5/94) homozygous recessive alleles. The high percentage of wild type individuals also suggests that amodiaquine is metabolized efficiently. For the Pfmdr1 gene, 95\% were wild type (N86) and 5\% mutant (Y86); 36\% were wild type (Y184), 64\% mutant (F184); $100 \%$ were wild type (D1246). High prevalence of N86, F184, and D1246 suggests AL is less efficacious than AA.

\section{Declarations}

Ethics approval and consent to participate

Ethical clearance was obtained from the Noguchi Memorial Institute for Medical Research (NMIMR) Institutional Review Board (IRB) and the Ghana Health Service IRB number IRB00001276. Informed consent was obtained from the guardians of the participants. This was after the purpose of the study has been explained to them in their native language and given an opportunity to ask questions that is of concern to them.

\section{Consent for publication}

Not applicable

Availability of data and materials

All data generated or analyzed during this study are included in this published article.

Competing interests

The authors declare that they have no competing interests

\section{Funding}

This research work was funded with grant offered to Peter Hodoameda by West African Centre for Cell Biology of Infectious Pathogens as part of his Mphil Fellowship. 
$\mathrm{PH}, \mathrm{NDQ}$, and NBQ conceived and designed the study. PH carried out the molecular genetic studies and led the drafting of the manuscript. NDQ, and NBQ also contributed to drafting of the manuscript. $\mathrm{OCH}$, SM, BA, and KK gave technical support and contributed significantly to drafting of the manuscript. All authors read and approved the final version of the manuscript.

\section{Acknowledgments}

The authors wish to acknowledge the contributions of Dr. Edmund Tetteh Nartey of the Centre for Tropical Clinical Pharmacology and Therapeutics (CTCPT), Mr. Samuel Ahorhorlu (CTCPT), Dr. William Kudzi (CTCPT) Mr. Phillip Agyeman (NMIMR), Miss Selassi Bruku (NMIMR). Nana Aba Enulson (NMIMR), Mr. Kofi Ntiamoah (NMIMR).

\section{References}

1. Abuaku, B., Duah, N., Quaye, L., Quashie, N., Malm, K., \& Plange, C. B. (2016). Therapeutic efficacy of artesunate - amodiaquine and artemether - lumefantrine combinations in the treatment of uncomplicated malaria in two ecological zones in Ghana. Malaria Journal, 4-11. https://doi.org/10.1186/s12936-015-1080-x

2. Bopp, S., Magistrado, P., Wong, W., Schaffner, S. F., Mukherjee, A., Lim, P., ... Volkman, S. K. (2018). Plasmepsin II-III copy number accounts for bimodal piperaquine resistance among Cambodian Plasmodium falciparum. Nature Communications, 9(1). https://doi.org/10.1038/s41467-018-04104Z

3. Cavaco, I., Stromberg-Norklit, J., Kaneko, A., Msellem, M. I., Dahoma, M., Ribeiro, V. L., ... Gil, J. P. (2005). CYP2C8 polymorphism frequencies among malaria patients in Zanzibar. 2, 15-18. https://doi.org/10.1007/s00228-004-0871-8

4. Chen, N., Chavchich, M., Peters, J. M., Kyle, D. E., Gatton, M. L., \& Cheng, Q. (2010). Deamplification of pfmdr1-containing amplicon on chromosome 5 in Plasmodium falciparum is associated with reduced resistance to artelinic acid in vitro. Antimicrobial Agents and Chemotherapy, 54(8), 33953401. https://doi.org/10.1128/AAC.01421-09

5. Duah, N. O., Matrevi, S. A., De Souza, D. K., Binnah, D. D., Tamakloe, M. M., Opoku, V. S., ... Koram, K. A. (2013). Increased pfmdr1 gene copy number and the decline in pfcrt and pfmdr1 resistance alleles in Ghanaian Plasmodium falciparum isolates after the change of anti-malarial drug treatment policy. Malaria Journal, 11, 135. https://doi.org/10.1186/1475-2875-11-135

6. Ferreira, P. E., Holmgren, G., Veiga, M. I., Uhlén, P., Kaneko, A., \& Gil, J. P. (2011). PfMDR1: Mechanisms of transport modulation by functional polymorphisms. PLOS ONE, 6(9), 3-10. https://doi.org/10.1371/journal.pone.0023875

7. Hodel, E. M., Ley, S. D., Qi, W., Ariey, F., Genton, B., \& Beck, H. (2009). A microarray-based system for the simultaneous analysis of single nucleotide polymorphisms in human genes involved in the metabolism of anti-malarial drugs. 9, 1-9. https://doi.org/10.1186/1475-2875-8-285 
8. Holmgren, G., Hamrin, J., Svärd, J., Mårtensson, A., Gil, J. P., \& Björkman, A. (2007). Selection of pfmdr1 mutations after amodiaquine monotherapy and amodiaquine plus artemisinin combination therapy in East Africa. Infection, Genetics and Evolution, 7(5), 562-569. https://doi.org/10.1016/j.meegid.2007.03.005

9. Ingelman-Sundberg, M., \& Rodriguez-Antona, C. (2005). Pharmacogenetics of drug-metabolizing enzymes: Implications for a safer and more effective drug therapy. Philosophical Transactions of the Royal Society B: Biological Sciences, 360(1460), 1563-1570. https://doi.org/10.1098/rstb.2005.1685

10. Kebamo, S., Tesema, S., \& Geleta, B. (2015). Journal of Drug Metabolism and The Role of Biotransformation in Drug Discovery and Development. 6(5). https://doi.org/10.4172/21577609.1000196

11. Kiaco, K., Rodrigues, A. S., Rosário, V., Gil, J. P., \& Lopes, D. (2017). The drug transporter ABCB1 c . $3435 \mathrm{C}>\mathrm{T}$ SNP influences artemether - lumefantrine treatment outcome. Malaria Journal, 1-6. https://doi.org/10.1186/s12936-017-2006-6

12. Kudzi, W., Dodoo, A. N. O., \& Mills, J. J. (2009). Characterisation of CYP2C8, CYP2C9 and CYP2C19 polymorphisms in a Ghanaian population. BMC Medical Genetics, 10, 8-13. https://doi.org/10.1186/1471-2350-10-124

13. Kudzi, W., Dodoo, A. N. O., \& Mills, J. J. (2010). Genetic polymorphisms in MDR1 , CYP3A4 and CYP3A5 genes in a Ghanaian population: a plausible explanation for altered metabolism of ivermectin in humans?

14. Lamba, J. K., Lin, Y. S., Schuetz, E. G., \& Thummel, K. E. (2012). Genetic contribution to variable human CYP3A-mediated metabolism. Advanced Drug Delivery Reviews, 64(SUPPL.), 256-269. https://doi.org/10.1016/j.addr.2012.09.017

15. Lee, T. M. N., Huang, L., Johnson, M. K., Lizak, P., Kroetz, D., Aweeka, F., \& Parikh, S. (2012). In vitro metabolism of piperaquine is primarily mediated by CYP3A4. Xenobiotica, 42(11), 1088-1095. https://doi.org/10.3109/00498254.2012.693972

16. Lef??vre, G., \& Thomsen, M. S. (1999). Clinical Pharmacokinetics of Artemether and Lumefantrine (Riamet??). Clinical Drug Investigation, 18(6), 467-480. https://doi.org/10.2165/00044011199918060-00006

17. Li, X.-Q., Björkman, A., Andersson, T. B., Ridderström, M., \& Masimirembwa, C. M. (2002). Amodiaquine clearance and its metabolism to $\mathrm{N}$-desethylamodiaquine is mediated by CYP2C8: a new high affinity and turnover enzyme-specific probe substrate. The Journal of Pharmacology and Experimental Therapeutics, 300(2), 399-407. Retrieved from http://www.ncbi.nlm.nih.gov/pubmed/11805197

18. Ministry of Health. (2009). Antimalarial Drug Policy for Ghana.

19. Mutagonda, R. F., Kamuhabwa, A. A. R., Minzi, O. M. S., Massawe, S. N., Asghar, M., Homann, M. V., ... Aklillu, E. (2017). Effect of pharmacogenetics on plasma lumefantrine pharmacokinetics and malaria 
treatment outcome in pregnant women. Malaria Journal, 16(1), 1-10.

https://doi.org/10.1186/s12936-017-1914-9

20. Ouji, M., Augereau, J.-M., Paloque, L., \& Benoit-Vical, F. (2018). Plasmodium falciparum resistance to artemisinin-based combination therapies: A sword of Damocles in the path toward malaria elimination. Parasite, 25, 24. https://doi.org/10.1051/parasite/2018021

21. Parikh, S., Ouedraogo, J. B., Goldstein, J. A., Rosenthal, P. J., \& Kroetz, D. L. (2007). Amodiaquine metabolism is impaired by common polymorphisms in CYP2C8: Implications for malaria treatment in Africa. Clinical Pharmacology and Therapeutics, 82(2), 197-203.

https://doi.org/10.1038/sj.clpt.6100122

22. Sá, J. M., Twu, O., Hayton, K., Reyes, S., Fay, M. P., Ringwald, P., \& Wellems, T. E. (2009). Geographic patterns of Plasmodium falciparum drug resistance distinguished by differential responses to amodiaquine and chloroquine . Proceedings of the National Academy of Sciences, 106(45), 1888318889. https://doi.org/10.1073/pnas.0911317106

23. Sisowath, C., Ferreira, P. E., Bustamante, L. Y., Dahlström, S., Mårtensson, A., Björkman, A., ... Gil, J. P. (2007). The role of pfmdr1 in Plasmodium falciparum tolerance to artemether-lumefantrine in Africa. Tropical Medicine and International Health, 12(6), 736-742. https://doi.org/10.1111/j.13653156.2007.01843.x

24. Vinayak, S., Alam, T., Sem, R., Shah, N. K., Susanti, A. I., Lim, P., ... Udhayakumar, V. (2010). Multiple Genetic Backgrounds of the Amplified Plasmodium falciparum Multidrug Resistance (pfmdr1) Gene and Selective Sweep of 184F Mutation in Cambodia. J Infect Dis., 201(10), 1551-1560. https://doi.org/10.1086/651949

25. White, N. J., \& Nosten, F. (2007). Artemisinin-Based Combination Treatment of Falciparum Malaria. The American Journal of Tropical Medicine and Hygiene, 77(6_Suppl), 181-192. https://doi.org/10.4269/ajtmh.2007.77.181

26. World Health Organization. (2014). World malaria report 2014. Retrieved April 23, 2019, from https://www.who.int/malaria/publications/world_malaria_report_2014/wmr-2014-no-profiles.pdf

27. World Health Organization. (2018). World Malaria Report 2018 Isbn 978924156565 3. Retrieved from www.who.int/malaria

28. Wu, A. H. B. (2011). Drug metabolizing enzyme activities versus genetic variances for drug of clinical pharmacogenomic relevance. Clinical Proteomics, 8(1), 1-9. https://doi.org/10.1186/1559-0275-812

29. Zanger, U. M., \& Schwab, M. (2013). Cytochrome P450 enzymes in drug metabolism: Regulation of gene expression, enzyme activities, and impact of genetic variation. Pharmacology and Therapeutics, 138(1), 103-141. https://doi.org/10.1016/j.pharmthera.2012.12.007

\section{Figures}




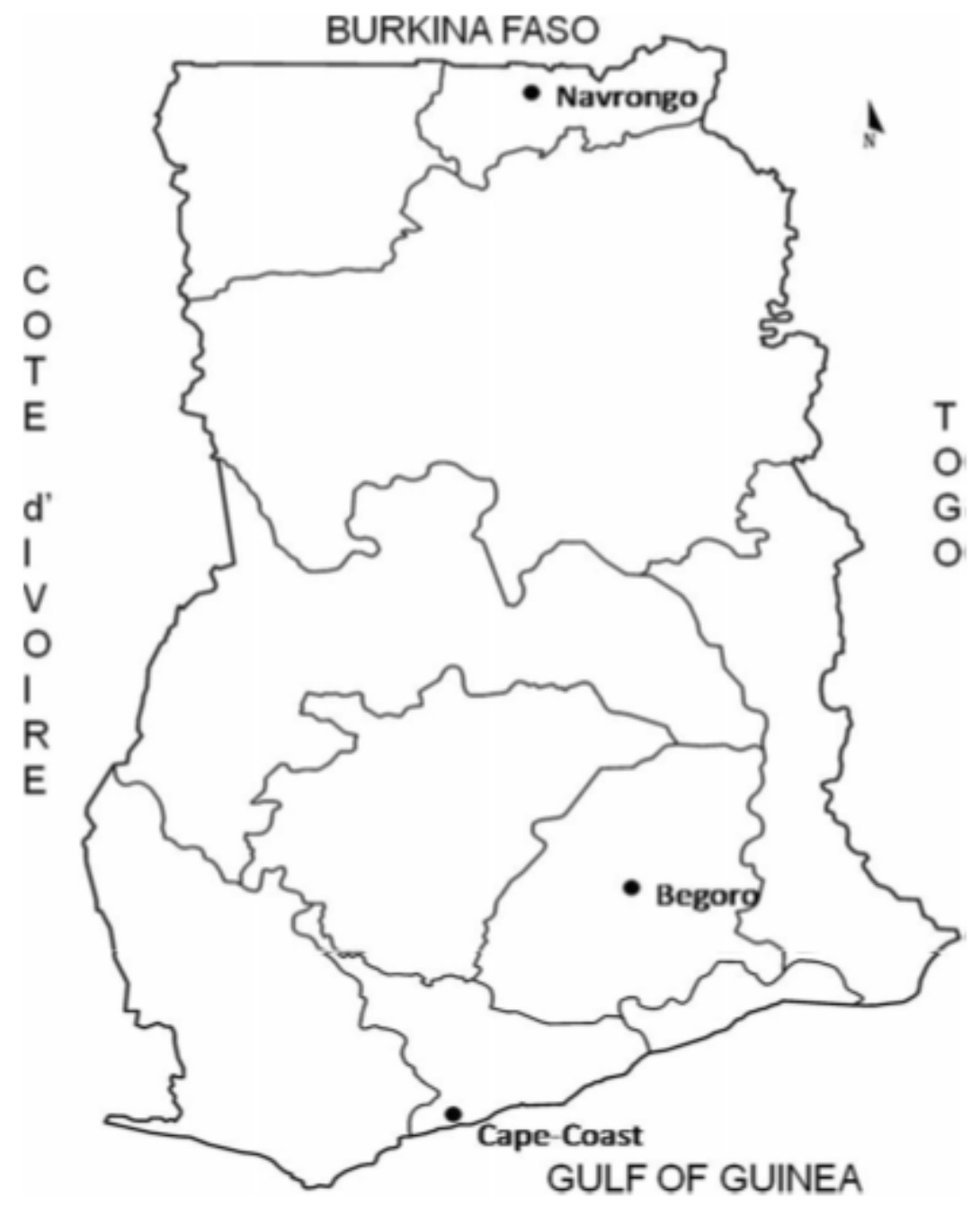

Figure 1

A map of Ghana. Showing the location of the study sites used in this study: Cape Coast, Begoro and Navrongo 
A

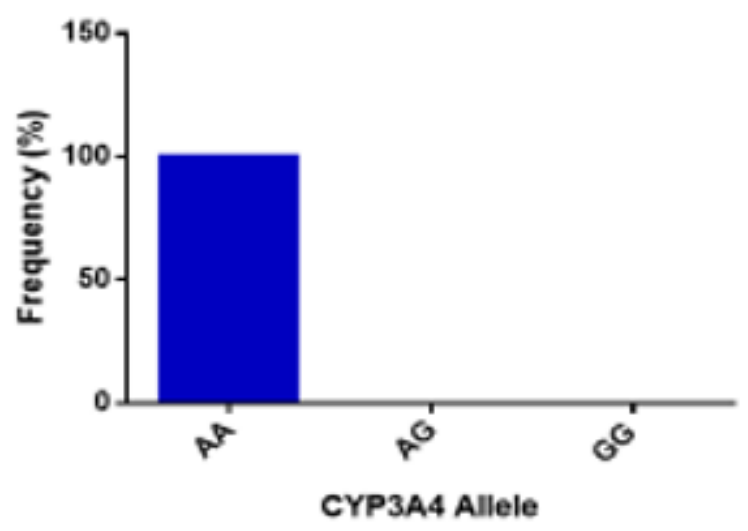

B

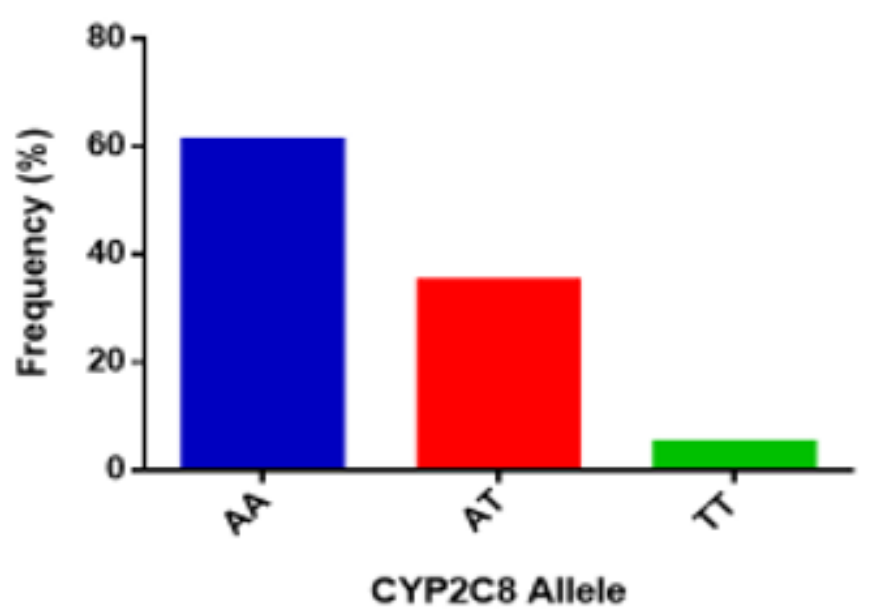

Figure 2

Prevalence of CYP3A4 and CYP2C8. (A) Genotype for CYP3A4 and (B) genotype for CYP2C8
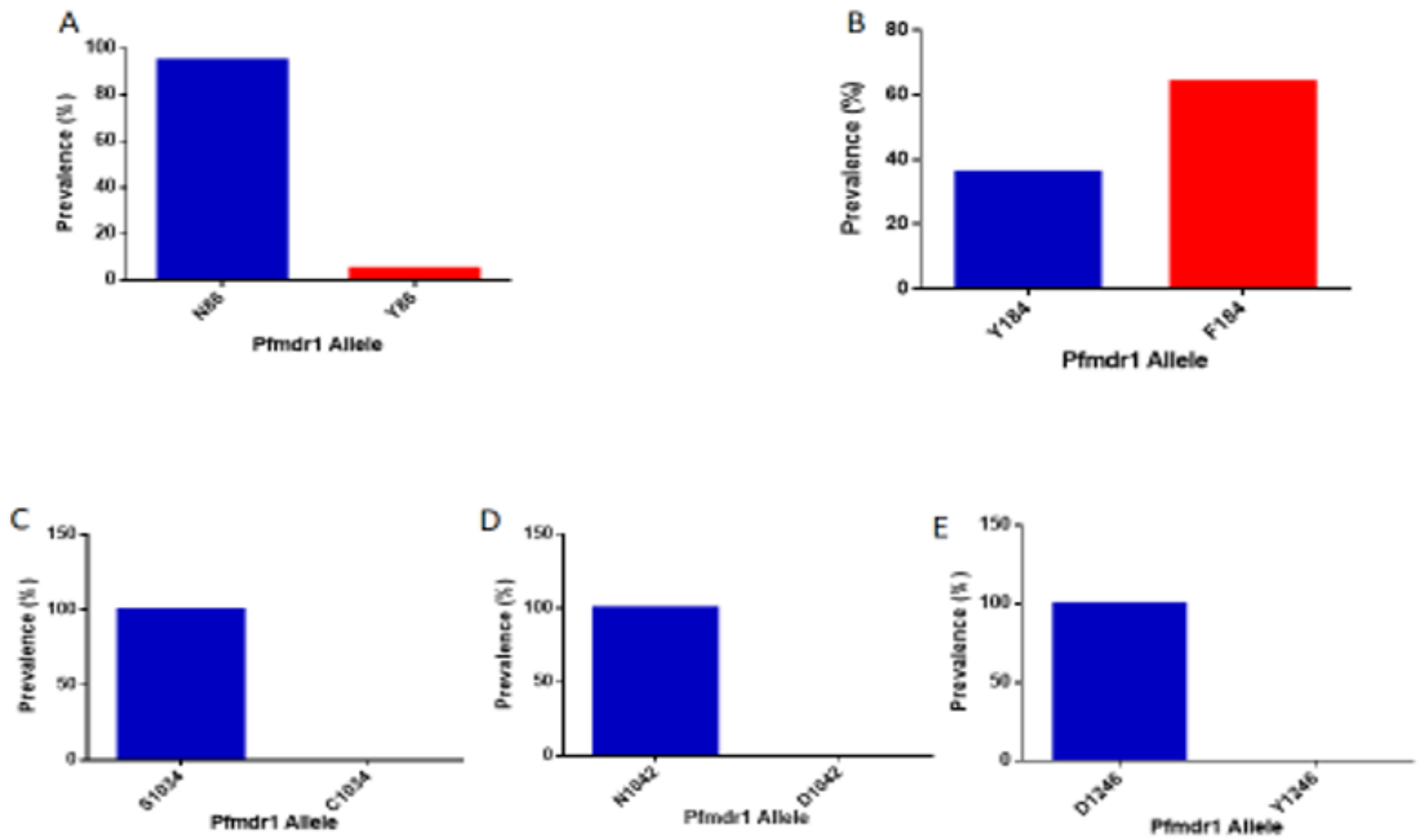

Figure 3 
Prevalence of Pfmdr1 codons 86, 184, 1034, 1042 and 1246. (A) Pfmdr1 86, (B) Pfmdr1 184, (C) Pfmdr1 1034, (D) Pfmdr1 1042 and (E) Pfmdr1 1246.

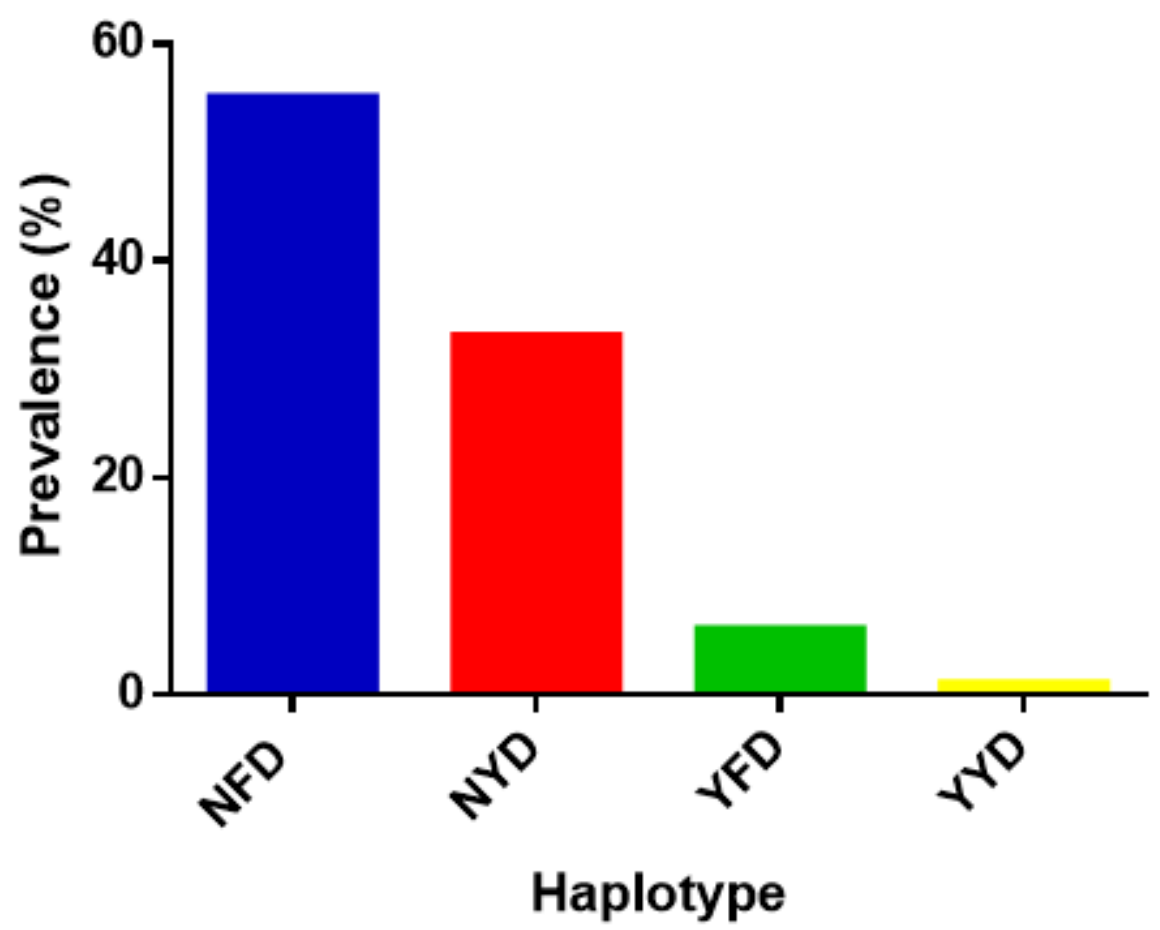

Figure 4

Prevalence of Pfmdr1 haplotype 